\title{
Angular anisotropy parameters for photoionization delays
}

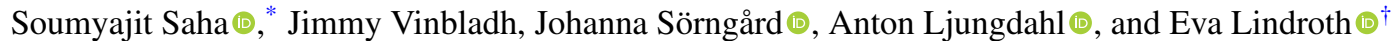 \\ Department of Physics, Stockholm University, AlbaNova University Center, SE-106 91 Stockholm, Sweden
}

(Received 7 July 2021; accepted 26 August 2021; published 15 September 2021)

\begin{abstract}
Anisotropy parameters describing the angular dependence of the photoionization delay are defined. The formalism is applied to results obtained with the relativistic random phase approximation with exchange for photoionization delay from the outermost $s$-orbitals in selected rare-gas atoms. Any angular dependence in the Wigner delay is induced here by relativistic effects, while the measurable atomic delay exhibits such a dependence also in the nonrelativistic limit. The contributions to the anisotropy from the different sources are disentangled and discussed. For the heavier rare gases, it is shown that measurements of the delay for electrons ejected in specific angles, relative to, e.g., those ejected along the laser polarization, are directly related here to the Wigner delay. For a considerable range of angles, the contributions from the second photon largely get canceled when the results in different angles are compared, and this angle-relative atomic delay is then close to the corresponding Wigner delay.
\end{abstract}

DOI: 10.1103/PhysRevA.104.033108

\section{INTRODUCTION}

The angular distribution of electrons released in the photoionization process is conveniently described with the angular anisotropy parameters. Originally introduced by Cooper and Zare [1] in the 1960s, the parameters have since emerged as the tool of choice to quantify and understand the photoionization angular distribution. The parametrization gives the photoelectron angular distribution after (multi)photon ionization with linearly polarized light as

$$
\frac{d \sigma}{d \Omega}=\frac{\sigma_{\text {int }}}{4 \pi}\left(1+\sum_{n=1}^{\infty} \beta_{n} P_{n}(\cos \theta)\right),
$$

where $P_{n}$ are Legendre polynomials, $\beta_{n}$ are the so-called asymmetry parameters, $\theta$ is the ejection angle with respect to the polarization axis, and $\sigma_{\text {int }}$ is the total cross section, i.e., the cross section integrated over all angles. For pure dipole transitions, only the even $n$ 's come into play, and when $k$ photons take part in the interaction, the sum terminates at $n=$ $2 k$. In-depth discussions on the parametrization, including the extension to unpolarized and differently polarized light, can, for example, be found in Refs. [2-4].

The angular asymmetry is sensitive to the relative phase shifts of the photoelectron partial waves, and it has long been used to obtain phase information. More recently, the development of attosecond techniques based on laser-assisted

\footnotetext{
*soumyajit.saha@fysik.su.se

†Eva.Lindroth@fysik.su.se
}

Published by the American Physical Society under the terms of the Creative Commons Attribution 4.0 International license. Further distribution of this work must maintain attribution to the author(s) and the published article's title, journal citation, and DOI. Funded by Bibsam. photoionization, such as the reconstruction of attosecond beating by interference of two-photon transitions (RABBIT) [5] or the attosecond streak camera [6], has provided an additional path to such information [7-15]. In these measurements, ionization, typically by extreme ultraviolet (XUV) light, is taking place in the presence of an infrared (IR) laser field, phase-locked to the XUV field. This paves the way for stimulated emission and absorption of IR photons, and sidebands appear in the photoelectron spectrum. Interference between the IR-absorption and -emission paths results in sideband modulations, directly depending on the relative phases acquired along the different routes. The sideband modulations are further angular-dependent. Absolute phase information is not accessible with these techniques either, but phase shifts between electrons originating from different states within the same atom $[7,8]$, or from different atoms [10-12,16], are readily available. Also, a specific angle can be used as a reference: in Refs. $[15,17]$, the relative phases of electron wave packets ejected in different angles were measured relative to those emitted along the direction of polarization of the laser field. The phase shift is related to the time it takes for an electron to escape an atomic potential [18-20] and is often presented in the form of a photoionization delay. The delay measured in the RABBIT experiments is usually called the atomic delay, and it involves always the exchange of at least two photons, while the underlying more fundamental delay in the one-photon ionization process is labeled the Eisenbud [18]-Wigner [19]-Smith [20] delay (or just Wigner delay for short).

Several aspects of laser-assisted photoionization exhibit an angular dependence: The photoelectron angular distribution (PAD) depends not only on the number and energy of the photons exchanged in the process, but also on the delay between the attosecond pulse train and the probe field, as recently discussed by Busto et al. [21], and further elaborated on by Joseph et al. [22]. In addition, the photoionization delay varies 
with detection angle. Here we will focus on the latter aspect. In Sec. II we will show that anisotropy parameters can be defined which describe the angular dependence of the delay. The atomic delay then takes a form similar to the corresponding case in Eq. (1):

$$
\tau_{A}(\theta)=\tau_{A}^{\mathrm{int}}+\frac{1}{2 \omega} \arg \left(1+\sum_{n=1}^{n_{\max }} \tilde{\beta}_{2 n} P_{2 n}(\cos \theta)\right),
$$

where $n_{\max }=2$ if a pure two-photon process is considered, and $\tau_{A}^{\mathrm{int}}$ is the delay that is measured with a detector that collects electrons emitted in all angles [23]. The laser frequency is given by $\omega$, and linearly polarized light as well as the validity of the dipole approximation are assumed. The new $\beta$-parameters, which we will call $\tilde{\beta}$, are closely related to the ordinary parameters in Eq. (1), but they are complex.

In Sec. III we apply the formalism to photoionization delay from the outermost $s$-orbital in argon, krypton, and xenon exposed to linearly polarized light. In a nonrelativistic description, the one-photon electron emission from an $s$-orbital consists of a single channel. The photoelectron is then described just by a $p$-wave; the $\beta_{2}$-parameter is consequently independent of energy, in this case $\beta_{2}=2$, and the corresponding photoionization delay (the Wigner delay) is isotropic. The two-photon amplitudes, on the other hand, have an angular dependence that varies with the photoelectron energy. This dependence arises due to the presence of two continua, in this case of $s$ - and $d$-character. Consequently, the atomic delay, cf. Sec. II B below, has an intrinsic angular dependence. That this indeed is a measurable effect was shown experimentally for helium in Ref. [17]. However, in a relativistic context we should expect the presence of two channels for ionization of an $s$-orbital: $s \rightarrow p_{1 / 2}, p_{3 / 2}$, to allow for an angular dependence of the delay, which is then of relativistic origin, already at the one-photon level. This latter case has been discussed by Kheifets et al. [24], who presented Wigner delays for electrons emitted from the outermost $s$-orbital in rare gases. Here we calculate the two-photon amplitudes and quantify different contributions to the angular dependence of the experimentally accessible atomic delay.

\section{THEORY}

\section{A. Two-photon matrix elements in a nonrelativistic framework}

The spatial part of the photoelectron wave function can be expressed as a sum over the contributing angular momentum channels. Assuming a nonrelativistic framework, we have

$$
\Psi_{\epsilon}(r, \theta, \phi)=\sum_{\ell, m} u_{\ell}(r) Y_{\ell m}(\theta, \phi)
$$

At large distances from the remaining ion, the radial channel functions are outgoing phase-shifted Coulomb waves that asymptotically approach plane waves. As discussed in Sec. II B below, we need to consider the interaction with two photons, $\Omega$ (with an XUV frequency) and $\omega$ (with an IR frequency), and write the asymptotic radial functions after interaction with both photons as

$$
\lim _{r \rightarrow \infty} u_{\ell}^{(2)}(r)=-\pi M^{(2)}(f, \omega, \Omega, 0) \sqrt{\frac{2 m}{\pi k \hbar^{2}}} e^{i\left(k r+\eta \ln 2 k r+\Delta_{\ell}\right)},
$$

where $M^{(2)}(f, \omega, \Omega, 0)$ is the complex two-photon matrix element, which connects the initial state, 0 , with the final state, $f$, where one electron with angular momentum $\ell$ has been released into the continuum. The superscript denotes that two photons are exchanged. The ionized electron has momentum $k$, and $\eta=Z / k a_{0}$, with $a_{0}$ being the Bohr radius and $Z$ is the charge of the remaining ion. The phase shift, $\Delta_{\ell}$, is

$$
\Delta_{\ell}=-\ell \frac{\pi}{2}+\sigma_{\eta, \ell}+\delta_{k, \ell}
$$

with the Coulomb phase

$$
\sigma_{\eta, \ell}=\arg [\Gamma(\ell+1-i \eta)]
$$

and a possible additional phase shift, $\delta_{k, \ell}$, induced by the atomic many-body potential at short distances from the nucleus.

Since the IR photon cannot by itself ionize the atom, the dominating contribution to $M^{(2)}$ comes from the time order where the XUV photon first releases the electron into the continuum, and there it subsequently interacts with the laser field by emitting or absorbing a laser photon. The contribution from the reversed time-order (i.e., when the laser photon is absorbed or emitted first and the XUV photon later) has been shown to be insignificant as long as the laser-matter interaction is expressed in length gauge [25], and only the dominating time-order will be treated here.

For this dominating time-order, and if electron-correlation is neglected, the two-photon matrix element that determines the amplitude for the transfer of an electron from orbital $a$ to the continuum state $q$ is

$$
M_{a \rightarrow q}^{(2)}=\frac{1}{i} E_{\omega} E_{\Omega} \lim _{\varepsilon \rightarrow 0^{+}} \sum_{s} \int \frac{\langle q|e \mathbf{r}| s\rangle\langle s|e \mathbf{r}| a\rangle}{\epsilon_{a}+\Omega-\epsilon_{s}+i \varepsilon} .
$$

The sum over intermediate states $s$ runs over all available states both bound and in the continuum. Here we use the random phase approximation with exchange (RPAE) [2] to include important correlation effects.

RPAE accounts fully for hole-particle excitations, including interchannel coupling and so-called ground-state correlation, and it is well established that the method is adequate to calculate (one-photon) photoionization cross sections in regions free of narrow resonances. In particular, it is able to describe the pronounced cross-section modulations that are known under the name Cooper minima, which will be discussed in Sec. III below. However, RPAE does not account for double holes and thus not for shake-up or shake-off processes where photoionization from a particular orbital is accompanied by excitation or ionization from other orbitals. Such effects are typically seen as sharp features in photoionization spectra.

Assuming that the interaction with the ionizing XUVphoton is well enough described within RPAE, we can calculate the two-photon matrix element as the transition matrix element from the RPAE-level perturbed wave function to 
the final continuum state. Exterior complex scaling is used in order to be able to use a finite numerical box, and the integration is performed numerically out to a distance far outside the atomic core, but within the unscaled region, while the last part of the integral is carried out using analytical Coulomb waves along the imaginary radial axis. The amplitude and phase shift of these Coulomb waves are determined from the numerical solutions for the perturbed wave function, as well as from the numerical description of a free electron in the potential of the remaining ion. How this is done, and how the numerical accuracy is monitored, has been discussed in quite some detail elsewhere [25-27].

\section{B. Sideband modulations}

We will restrict the discussion here to RABBIT experiments. This technique is performed with a train of attosecond pulses, i.e., an XUV comb in the frequency domain, which is assisted by a weak laser field, and, as discussed, e.g., in Ref. [14], it can combine attosecond temporal resolution with sub-eV spectral resolution. The RABBIT signal can be understood and quantified from the pure two-photon contribution: The ionization process is initiated by the XUV comb (the teeth are all odd multiples of the driving laser field), and sidebands are formed when an additional IR photon is either absorbed or emitted. The absorption path from one tooth in the XUV spectrum will then result in the same photoelectron energy, as the next tooth accompanied by IR emission. The intensity of a RABBIT sideband can be written as [28,29]

$$
\begin{aligned}
S & =\left|A_{a}+A_{e}\right|^{2}=\left|A_{a}\right|^{2}+\left|A_{e}\right|^{2}+A_{a}^{*} A_{e}+A_{a} A_{e}^{*} \\
& =\left|A_{a}\right|^{2}+\left|A_{e}\right|^{2}+2 \times\left|A_{a}\right|\left|A_{e}\right| \cos \left[\arg \left(A_{a}\right)-\arg \left(A_{e}\right)\right],
\end{aligned}
$$

where $A_{a / e}$ are the complex quantum amplitudes for the twophoton processes involving absorption (a) or emission (e) of an IR photon. The last term in Eq. (8) gives rise to oscillations in the sideband intensity, originating from the phase difference of the electron wave packets reaching the sideband through the two paths. It can be shown [29] that this phase difference has three sources: the delay between the IR and XUV pulses $(\tau)$, the group delay of the attosecond pulses ( $\left.\tau_{\mathrm{XUV}}\right)$, and the ionization process, which depends on the particular atomic $\operatorname{system}\left(\eta_{A}\right)$ :

$$
\cos \left[\arg \left(A_{a}\right)-\arg \left(A_{e}\right)\right]=\cos \left[2 \omega\left(\tau-\tau_{\mathrm{XUV}}\right)-\eta_{A}\right] .
$$

The latter can be interpreted as an atomic delay: $\tau_{A}=\eta_{A} / 2 \omega$. Since the delay between the two light fields is controlled in experiments and the light field group delay can be canceled through relative measurements, the atomic contribution can be extracted. In principle, absorption or emission of additional IR photons can contribute to the sideband signal, which would complicate the analysis. Experiments are therefore generally performed with a weak IR field, and in addition Fourier techniques can be used to extract the $2 \omega$ oscillation [12,23,29], which is due to the pure two-photon signal. It is thus the two-photon matrix elements, cf. Eq. (7), that are needed for the theoretical modeling. A recent review of the experimental method can be found in Ref. [29].

The quantity in Eq. (8) is of course real, but the individual terms

$$
A_{e}^{*} A_{a}=\left(A_{e} A_{a}^{*}\right)^{*}=\left|A_{a}\right|\left|A_{e}\right| e^{i\left[\arg \left(A_{a}\right)-\arg \left(A_{e}\right)\right]}
$$

are complex, and it is their argument that determines the sideband modulations.

\section{Anisotropy parameters}

The angular dependence of the photoelectron emission is governed by the relative strengths of the different angular momentum channels. Using Eqs. (3) and (4), and omitting the factors common to all channels (which cannot affect the relative strength), we write the amplitude that governs the angular dependence as

$$
A(\theta, \phi)=\sum_{\ell, m, m_{s}} Y_{\ell m}(\theta, \phi) e^{i \Delta_{\ell}} M_{n_{a} \ell_{a} m m_{s} \rightarrow \ell m m_{s}}^{(2)} .
$$

Here linearly polarized light and emission from a closed-shell orbital, defined by $n_{a} \ell_{a}$, are assumed. The azimuthal quantum number, as well as the spin direction, of the photoelectron, will, with linear polarization, be the same as the orbital from which it emerged. The modulus square of the amplitude in Eq. (11) will under these circumstances be independent of the azimuthal angle, i.e., there will be no $\phi$-dependence.

Angular momentum coupling theory can be used to rewrite $M^{(2)}$ as a reduced matrix element that is independent of the azimuthal quantum numbers. This reduced matrix element consists of effective two-photon operators of rank 0,1 , and 2 , and a $3 j$-symbol (cf. the Appendix). For linearly polarized light the rank-1 operator does not contribute, and it is enough to consider the operators of rank 0 and 2 . The modulus square of the amplitude is now

$$
|A(\theta)|^{2}=\sum_{m, m_{s}}\left|\sum_{K, \ell} Y_{\ell m}(\theta, 0)(-1)^{\ell-m}\left(\begin{array}{ccc}
\ell & K & \ell_{a} \\
-m & 0 & m
\end{array}\right) e^{i \Delta_{\ell}} \mathbf{M}_{\ell, K}^{(2)}\right|^{2},
$$

where $\mathbf{M}_{\ell, K}^{(2)}$ is the reduced two-photon matrix element on the right-hand side of Eq. (A2) [note the difference from the quantity $\mathbf{M}^{(2)}$ in Eq. (11)]. Since the modulus square of the amplitude lacks $\phi$-dependence, the spherical harmonics can be evaluated at $\phi=0$. The subscript $\ell$ denotes the angular momen- tum of the photoelectron, and $K$ is the total rank of the twophoton interaction. The cross terms in Eq. (12) will depend on the relative phase shift of the different angular momentum channels, as well as on the phase of the matrix element, and hereby the angular distribution depends on these phases. 
The standard two-photon cross section, i.e., with a single set of photon energies $\left(\Omega_{1}, \Omega_{2}\right)$, involves only one path to the final energy state, e.g., the absorption path with final energy $E_{0}+\Omega_{1}+\Omega_{2}$. The $\beta$-parameters in Eq. (1) will then be expressed in terms of the contributing $e^{i \Delta_{\ell}} \mathbf{M}_{\ell, K}^{(2)}$ (specific for each quantum system) and numerical constants, common to ionization from a particular orbital angular momentum. For example, for ionization from an $s$-orbital the parameters are

$$
\begin{aligned}
\beta_{2}= & \frac{\frac{2}{7}\left|\mathbf{M}_{2,2}^{(2)}\right|^{2}}{\frac{1}{5}\left|\mathbf{M}_{2,2}^{(2)}\right|^{2}+\left|\mathbf{M}_{0,0}^{(2)}\right|^{2}} \\
& +\frac{\left[\left(\mathrm{M}_{0,0}^{(2)}\right)^{*} \mathbf{M}_{2,2}^{(2)} e^{i\left(\Delta_{2}-\Delta_{0}\right)}+\mathrm{M}_{0,0}^{(2)}\left(\mathrm{M}_{2,2}^{(2)}\right)^{*} e^{-i\left(\Delta_{2}-\Delta_{0}\right)}\right]}{\frac{1}{5}\left|\mathbf{M}_{2,2}^{(2)}\right|^{2}+\left|\mathbf{M}_{0,0}^{(2)}\right|^{2}}
\end{aligned}
$$

and

$$
\beta_{4}=\frac{\frac{18}{35}\left|\mathbf{M}_{2,2}^{(2)}\right|^{2}}{\frac{1}{5}\left|\mathbf{M}_{2,2}^{(2)}\right|^{2}+\left|\mathbf{M}_{0,0}^{(2)}\right|^{2}}
$$

while the amplitude integrated over all angles (i.e., the quantity proportional to the total cross section) is

$$
\int|A(\theta)|^{2} d \Omega=2\left(\frac{1}{5}\left|\mathbf{M}_{2,2}^{(2)}\right|^{2}+\left|\mathbf{M}_{0,0}^{(2)}\right|^{2}\right)
$$

In this particular case, $\mathbf{M}_{0,0}^{(2)}$ and $\mathbf{M}_{2,2}^{(2)}$ are the two-photon matrix elements for the $s \rightarrow p \rightarrow s$ and $s \rightarrow p \rightarrow d$ channels, respectively, and only the two-photon operator with $K=0$ can connect the bound $s$-orbital with an outgoing $s$-electron and only the $K=2$ operator can connect it with an outgoing $d$-electron. The factor of 2 comes from the sum over the spin-projections.

It is interesting to note that the photoelectron amplitude, perpendicular to the polarization direction, i.e., at $\theta=\pi / 2$, is 0 when $4 \beta_{2}-3 \beta_{4}=8$. For high kinetic energies the radial matrix elements for the two channels will approach each other, and with the angular integrals given in Eq. (A5) we can conclude that $\beta_{2} \rightarrow 20 / 7$ and $\beta_{4} \rightarrow 8 / 7$, and thus indeed $4 \beta_{2}-3 \beta_{4} \rightarrow 8$, when the kinetic energy of the photoelectron goes to infinity. Below, we will find that the $\beta$-parameters are close to these values also for modest kinetic energies for the studied cases of above-threshold ionization. This reflects the limited possibilities for the IR-photon to change the direction of the photoelectron, and it illustrates the range of validity of the so-called "soft-photon approximation" [30].

Consider now the situation with two interfering amplitudes as in Eq. (8). The diagonal terms $\left|A_{a}\right|^{2}$ and $\left|A_{e}\right|^{2}$ are real and will be of the form given in Eq. (12), and the parametrization will be with the same form of the $\beta$-parameters as in Eqs. (13) and (14), although the values of the matrix elements and the phases will be different in the absorption and the emission paths. The situation for the cross terms, responsible for the modulation of the sidebands when the delay, $\tau$, between the light fields is varied, is, however, different. This complex term will be

$$
A_{a}^{*}(\theta) A_{e}(\theta)=\sum_{m, m_{s}}\left[\sum_{\ell, K} Y_{\ell, m}(\theta, 0)(-1)^{\ell-m}\left(\begin{array}{ccc}
\ell & K & \ell_{a} \\
-m & 0 & m
\end{array}\right) e^{i \Delta_{\ell}} \mathbf{M}_{a, \ell, K}\right]^{*}\left[\sum_{\ell, K} Y_{\ell, m}(\theta, 0)(-1)^{\ell-m}\left(\begin{array}{ccc}
\ell & K & \ell_{a} \\
-m & 0 & m
\end{array}\right) e^{i \Delta_{\ell}} \mathbf{M}_{e, \ell, K}\right] \text {, }
$$

where it is understood that $\mathbf{M}_{a / e}$ denote the two-photon matrix elements for the two paths, and the superscript (2) has thus been dropped. We note that the form is exactly the same as in Eq. (12) and it can readily be shown that for ionization from an $s$-orbital the result is

$$
\begin{aligned}
A_{a}^{*}(\theta, 0) A_{e}(\theta, 0)= & \frac{1}{4 \pi}\left(\frac{1}{5} \mathbf{M}_{a, 2,2}^{*} \mathbf{M}_{e, 2,2}+\mathbf{M}_{a, 0,0}^{*} \mathbf{M}_{e, 0,0}\right) \\
& \times\left(1+\tilde{\beta}_{2} P_{2}(\cos \theta)+\tilde{\beta}_{4} P_{4}(\cos \theta)\right)
\end{aligned}
$$

with

$$
\begin{aligned}
\tilde{\beta}_{2}= & \frac{\frac{2}{7} \mathbf{M}_{a, 2,2}^{*} \mathbf{M}_{e, 2,2}}{\frac{1}{5} \mathbf{M}_{a, 2,2}^{*} \mathbf{M}_{e, 2,2}+\mathbf{M}_{a, 0,0}^{*} \mathbf{M}_{e, 0,0}} \\
& +\frac{\mathbf{M}_{a, 0,0}^{*} \mathbf{M}_{e, 2,2} e^{i\left(\Delta_{2}-\Delta_{0}\right)}+\mathbf{M}_{e, 0,0} \mathbf{M}_{a, 2,2}^{*} e^{-i\left(\Delta_{2}-\Delta_{0}\right)}}{\frac{1}{5} \mathbf{M}_{a, 2,2}^{*} \mathbf{M}_{e, 2,2}+\mathbf{M}_{a, 0,0}^{*} \mathbf{M}_{e, 0,0}}
\end{aligned}
$$

and

$$
\tilde{\beta}_{4}=\frac{\frac{18}{35} \mathbf{M}_{a 2,2}^{*} \mathbf{M}_{e 2,2}}{\frac{1}{5} \mathbf{M}_{a, 2,2}^{*} \mathbf{M}_{e, 2,2}+\mathbf{M}_{a, 0,0}^{*} \mathbf{M}_{e, 0,0}} .
$$

The numerical constants are the same as in Eqs. (13) and (14), but since the absorption and emission matrix elements are distinctly different, the $\tilde{\beta}$ 's are complex. The atomic delay can now be written as

$$
\begin{aligned}
\tau_{A}(\theta)= & \frac{\arg \left[\frac{1}{5} \mathbf{M}_{a, 2,2}^{*} \mathbf{M}_{e, 2,2}+\mathbf{M}_{a, 0,0}^{*} \mathbf{M}_{e, 0,0}\right]}{2 \omega} \\
& +\frac{\arg \left[\left(1+\tilde{\beta}_{2} P_{2}(\cos \theta)+\tilde{\beta}_{4} P_{4}(\cos \theta)\right)\right]}{2 \omega} \\
= & \tau_{A}^{\mathrm{int}}+\frac{\arg \left[\left(1+\tilde{\beta}_{2} P_{2}(\cos \theta)+\tilde{\beta}_{4} P_{4}(\cos \theta)\right)\right]}{2 \omega},
\end{aligned}
$$

where the delay that will be measured from an angular integrated signal, i.e., $\tau_{A}^{\text {int }}$, is recognized on the first line.

Finally, we want to emphasize that although the last factor in Eq. (17) gives unity after angular integration, there is nothing forcing the last term in Eq. (20) to integrate to zero: $\tau_{A}^{\text {int }}$ is 
the delay extractable from an angular integrated experiment, it is not $\tau_{A}(\theta)$ integrated over all angles.

\section{Connection to other forms of parametrization}

Before continuing, we note in passing that an alternative form of Eq. (17) is

$$
\begin{aligned}
A_{a}^{*}(\theta) A_{e}(\theta)= & \frac{1}{4 \pi}\left|\frac{1}{5} \mathrm{M}_{a, 2,2}^{*} \mathrm{M}_{e, 2,2}+\mathrm{M}_{a, 0,0}^{*} \mathrm{M}_{e, 0,0}\right| e^{i \phi_{0}} \\
& \times\left(1+\left|\tilde{\beta}_{2}\right| e^{i \phi_{20}} P_{2}(\cos \theta)+\left|\tilde{\beta}_{4}\right| e^{i \phi_{40}} P_{4}(\cos \theta)\right),
\end{aligned}
$$

where the phases are named by Joseph et al. [22]. Adding the complex conjugate, $A_{a}(\theta) A_{e}^{*}(\theta)$, one can directly connect to their parametrization. They write the sideband oscillation, cf. Eq. (9), as coming from three terms: one angular-independent term that oscillates as $\cos \left(\omega \tau+\phi_{0}\right)$, one term proportional to $P_{2}(\cos \theta)$ that oscillates as $\cos \left(\omega \tau+\phi_{0}+\phi_{20}\right)$, and finally one term proportional to $P_{4}(\cos \theta)$ that oscillates as $\cos \left(\omega \tau+\phi_{0}+\phi_{40}\right)$. The arguments of the complex $\tilde{\beta}$ 's are thus identical to the phases $\phi_{20}$ and $\phi_{40}$ in Ref. [22].

\section{E. Relativistic formalism}

In the relativistic case, the asymptotic radial wave function is a two-component function, with a phase shift slightly different compared to the nonrelativistic description $[31,32]$ :

$$
\begin{aligned}
\lim _{r \rightarrow \infty} u_{\kappa}^{(n)}(r)= & -\pi M^{(n)} \sqrt{\frac{1}{1+\zeta^{2}}} \sqrt{\frac{2 m}{\pi k \hbar^{2}}\left(1+\frac{\varepsilon}{m c^{2}}\right)} \\
& \times\left(\begin{array}{c}
e^{i\left(k r+\eta \ln 2 k r+\Delta_{\kappa}\right)} \\
\left.i \zeta e^{i\left(k r+\eta \ln 2 k r+\Delta_{\kappa}\right.}\right)
\end{array}\right) .
\end{aligned}
$$

Equation (22), which is valid after one photon $(n=1)$, as well as after two $(n=2)$ photons, replaces thus the expression in Eq. (4). The relation between the large (upper) and small (lower) components at infinity is

$$
\zeta=\sqrt{\frac{E-m c^{2}}{E+m c^{2}}}=\frac{k \hbar}{2 m c} \frac{1}{\left(1+\frac{\varepsilon}{2 m c^{2}}\right)},
$$

where $E=m c^{2}+\varepsilon$ is the energy including the rest mass contribution, and $k$, as well as $\eta$, are given by the relativistic generalizations of the nonrelativistic expressions:

$$
\begin{gathered}
k=\frac{\sqrt{E^{2}-m^{2} c^{4}}}{\hbar c}=\frac{\sqrt{2 \varepsilon m}}{\hbar} \sqrt{1+\frac{\varepsilon}{2 m c^{2}},} \\
\eta=Z \alpha E \sqrt{\frac{1}{E^{2}-m^{2} c^{4}}}=\frac{Z}{a_{0} k}\left(\frac{\varepsilon}{m c^{2}}+1\right) .
\end{gathered}
$$

The relativistic quantum number $\kappa$ is further defined as

$$
\begin{aligned}
& \kappa=-(\ell+1) \text { for } j=\ell+\frac{1}{2} \\
& \kappa=\ell \text { for } j=\ell-\frac{1}{2},
\end{aligned}
$$

where $\ell$ refers to the orbital angular momentum associated with the larger (upper) component of the wave function, cf. Eq. (28). The Coulomb phase shift is finally slightly different in the relativistic case and is given by

$$
\Delta_{\kappa}=-\gamma \pi / 2+\sigma_{\kappa}+v+\delta,
$$

where $\gamma^{2} \equiv \kappa^{2}-\alpha^{2} Z^{2}$ and

$$
\begin{gathered}
\sigma_{\kappa}=\arg [\Gamma(\gamma-i \eta)], \\
e^{2 i v}=\frac{\left(-\kappa+\frac{i Z}{k a_{0}}\right)}{(\gamma+i \eta)},
\end{gathered}
$$

while $\delta$, as before, is the shift induced by the short-range non-Coulombic potential, although now possibly adjusted by relativistic effects.

The two-component angular-dependent amplitudes, corresponding to the nonrelativistic expressions in Eq. (11), can thus be written as

$$
\begin{aligned}
A(\theta, \phi)= & \sum_{\kappa m_{j}} \sum_{K}(-1)^{j+K+j_{a}}\left[(-1)^{j-m_{j}}\left(\begin{array}{ccc}
j & K & j_{a} \\
-m_{j} & 0 & m_{j}
\end{array}\right)\right] \\
& \times\left(\begin{array}{c}
\sum_{m_{\ell_{f}} m_{s}}\left\langle\ell_{f} m_{\ell_{f}} s m_{s} \mid j m_{j}\right\rangle Y_{\ell_{f}, m_{\ell_{f}}}(\theta, \phi) \xi_{m_{s}} \\
i \zeta \sum_{m_{\ell_{g}} m_{s}}\left\langle\ell_{g} m_{\ell_{g}} s m_{s} \mid j m_{j}\right\rangle Y_{\ell_{g}, m_{\ell_{g}}}(\theta, \phi) \xi_{m_{s}}
\end{array}\right) \bar{M}_{\kappa, K}^{(n)},
\end{aligned}
$$

where $\xi_{ \pm}$(for $m_{s}= \pm 1 / 2$ ) denotes the spin function, and the angular momentum connected with the small (lower) component is $\ell_{g}=\ell_{f} \pm 1$, where $\ell_{f}$ refers to the angular momentum of the large (upper) component, $\ell_{f}=j \mp 1 / 2$. Further, we have $\left\langle\xi_{m_{s}} \mid \xi_{m_{s}^{\prime}}\right\rangle=\delta\left(m_{s}, m_{s}^{\prime}\right)$, and the notation $\bar{M}$ indicates that we now include the phase of the ionized electron, given in Eq. (26), cf. (A4) in the matrix element. Again, we may use Eq. (28) for both the one-photon contribution $(n=1, K=1)$ and the two-photon case $(n=2, K=0,2)$. The procedure to obtain the $\tilde{\beta}$ is sketched below, and the explicit forms for our test case of ionization from an $s$-orbital are given.

\section{The Wigner delay}

In the nonrelativistic framework, we calculate the onephoton contribution to the atomic delay as

$$
\tau_{W}(\epsilon)=\frac{\arg \left(A_{k_{>}}\right)-\arg \left(A_{k_{<}}\right)}{2 \omega},
$$

where $A$ is the complex one-photon amplitude for the emission $\left(k_{>}\right)$and the absorption arm $\left(k_{<}\right)$of the RABBIT paths. This coincides with the definition of the Wigner delay as the derivative of the phase shift with respect to energy within the finite-difference approximation. Since the relativistic amplitude has two radial components, with different angular parts, it might at first seem harder to define a single argument here. Remembering that the goal is to find the one-photon contribution to the atomic delay, we note that

$$
\begin{aligned}
\left|A_{a}+A_{e}\right|^{2} & =\left|A_{<}+\delta A_{a}+A_{>}+\delta A_{e}\right|^{2} \\
& =\left|A_{k_{<}}\right|^{2}+\left|A_{k_{>}}\right|^{2}+A_{k_{<}}^{*} A_{k_{>}}+A_{k_{>}}^{*} A_{k_{<}}+\cdots
\end{aligned}
$$

and conclude that the pure one-photon contribution to the RABBIT signal is given by the term

$A_{k_{<}}^{*} A_{k_{>}}+$c.c. $=2\left|A_{k_{<}}\right|\left|A_{k_{>}}\right| \cos \left(\arg \left(A_{k_{>}}\right)-\arg \left(A_{k_{<}}\right)\right)$,

where c.c. stands for complex conjugate. The argument of $A_{k_{<}}^{*} A_{k_{>}}$thus indeed agrees with the expression for $\tau_{W}$ in 
Eq. (29), but since the expression is now directly related to the modulus square of an amplitude, the argument can be unambiguously obtained.

Following the example from the nonrelativistic derivation, and the amplitude expression in Eq. (28), we write the angle-dependent amplitude, $A_{k_{<}}^{*}(\theta) A_{k_{>}}(\theta)$, as the integrated contribution times an angle-dependent factor. One-photon ionization from an $s$-orbital leads to $p_{1 / 2}(\kappa=1)$ and $p_{3 / 2}$ $(\kappa=-2)$ photoelectrons and the delay

$$
\begin{aligned}
\tau_{W}(\theta)= & \frac{1}{2 \omega} \arg \left[\overline{\mathbf{M}}_{\kappa=1}^{<, *} \overline{\mathbf{M}}_{\kappa=1}^{>}+\overline{\mathbf{M}}_{\kappa=-2}^{<, *} \overline{\mathbf{M}}_{\kappa=-2}^{>}\right] \\
& +\frac{1}{2 \omega} \arg \left[1+\tilde{\beta}_{2}^{W} P_{2}(\cos \theta)\right],
\end{aligned}
$$

where the first argument gives $\tau_{W}^{\text {int }}$, i.e., the one-photon contribution to the delay characterizing the sideband modulations of an angular integrated signal, and

$$
\tilde{\beta}_{2}^{W}=\frac{\overline{\mathbf{M}}_{\kappa=-2}^{<, *} \overline{\mathrm{M}}_{\kappa=-2}^{>}-\sqrt{2}\left(\overline{\mathrm{M}}_{\kappa=1}^{<, *} \overline{\mathrm{M}}_{\kappa=-2}^{>}+\overline{\mathrm{M}}_{\kappa=-2}^{<, *} \overline{\mathrm{M}}_{\kappa=1}^{>}\right)}{\overline{\mathbf{M}}_{\kappa=1}^{<, *} \overline{\mathbf{M}}_{\kappa=1}^{>}+\overline{\mathrm{M}}_{\kappa=-2}^{<, *} \overline{\mathrm{M}}_{\kappa=-2}^{>}} .
$$

\section{Atomic delay}

In the two-photon case, Eq. (28) gives, through the same procedure as above, the atomic delay as combinations of the matrix elements for ionization in the $s_{1 / 2}(\kappa=-1), d_{3 / 2}$ $(\kappa=2)$, and $d_{5 / 2}(\kappa=-3)$ channels. The first of these is due to the two-photon operator with rank 0 , and the last two are due to the two-photon operator with rank 2, and we have dropped the $K$-index in the following. The atomic delay from the integrated signal is

$$
\tau_{A}^{\text {int }}=\frac{1}{2 \omega} \arg [D]
$$

where

$$
\begin{aligned}
\mathrm{D}= & \overline{\mathrm{M}}_{a, \kappa=-1}^{*} \overline{\mathrm{M}}_{e, \kappa=-1} \\
& +\frac{1}{5} \overline{\mathrm{M}}_{a, \kappa=2}^{*} \overline{\mathrm{M}}_{e, \kappa=2}+\frac{1}{5} \overline{\mathrm{M}}_{a, \kappa=-3}^{*} \overline{\mathrm{M}}_{e, \kappa=-3},
\end{aligned}
$$

where, as in Eq. (16), it is understood that $\overline{\mathbf{M}}_{a / e}$ are two-photon matrix elements so that the superscript (2) can be dropped, and, as noted in connection with Eq. (28), the matrix elements include the phase of the outgoing electron, cf. (A4). The $\tilde{\beta}$ parameters are now

$$
\begin{aligned}
\tilde{\beta}_{2}= & \frac{1}{\mathrm{D}}\left\{\frac{1}{5}\left(\overline{\mathbf{M}}_{a, \kappa=2}^{*} \overline{\mathbf{M}}_{e, \kappa=2}+\frac{8}{7} \overline{\mathbf{M}}_{a, \kappa=-3}^{*} \overline{\mathbf{M}}_{e, \kappa=-3}\right)\right. \\
& +\frac{3}{\sqrt{15}}\left(\overline{\mathbf{M}}_{a, \kappa=-1}^{*} \overline{\mathbf{M}}_{e, \kappa=-3}+\overline{\mathbf{M}}_{a, \kappa=-3}^{*} \overline{\mathbf{M}}_{e, \kappa=-1}\right) \\
& +\frac{2}{\sqrt{10}}\left(\overline{\mathbf{M}}_{a, \kappa=-1}^{*} \overline{\mathbf{M}}_{e, \kappa=2}+\overline{\mathbf{M}}_{a, \kappa=2}^{*} \overline{\mathbf{M}}_{e, \kappa=-1}\right) \\
& \left.+\frac{2 \sqrt{\frac{3}{2}}\left(\overline{\mathbf{M}}_{a, \kappa=-3}^{*} \overline{\mathbf{M}}_{e, \kappa=2}+\overline{\mathbf{M}}_{a, \kappa=2}^{*} \overline{\mathbf{M}}_{e, \kappa=-3}\right)}{35}\right\}
\end{aligned}
$$

and

$$
\begin{aligned}
\tilde{\beta}_{4}= & \frac{1}{\mathrm{D}}\left\{\frac { 6 } { 3 5 } \left(\overline{\mathbf{M}}_{a, \kappa=-3}^{*} \overline{\mathbf{M}}_{e, \kappa=-3}\right.\right. \\
& \left.\left.+2 \sqrt{\frac{3}{2}}\left(\overline{\mathbf{M}}_{a, \kappa=-3}^{*} \overline{\mathbf{M}}_{e, \kappa=2}+\overline{\mathbf{M}}_{a, \kappa=2}^{*} \overline{\mathbf{M}}_{e, \kappa=-3}\right)\right)\right\}
\end{aligned}
$$

and now Eq. (2) can be used to get the angular-dependent delay.

\section{RESULTS AND DISCUSSION}

The calculations of the two-photon matrix elements have been done with a relativistic implementation [33] of the procedure described for nonrelativistic systems in Refs. [26,27]. The method is based on the random phase approximation with exchange.

The IR-photon energy is kept at $1.55 \mathrm{eV}$ in all the calculations. This corresponds to the wavelengths commonly used in RABBIT experiments.

\section{A. The delay from angular integrated measurements}

In experiments where electrons emitted in all directions are collected, the sideband modulations depend on the atomic delay $\tau_{A}^{\text {int }}$, cf. Eqs. (34) and (35), as $\cos \left(2 \omega\left(\tau+\tau_{\mathrm{XUV}}-\tau_{A}^{\mathrm{int}}\right)\right)$, cf. Eq. (9). This delay is shown in the top panel of Fig. 1 for ionization from the outermost $s$-shell in argon (solid line), krypton (dashed line), and xenon (dash-dotted line). The middle panel shows the underlying one-photon Wigner delay, $\tau_{W}^{\text {int }}$, cf. Eq. (32), while the bottom panel shows the difference between the two, i.e., the isolated contribution from the second photon. The label (cc) in the bottom panels stands for continuum-continuum delay.

All the systems displayed in Fig. 1 are known to have a so-called Cooper minimum [34] in the cross section from the outermost orbital (which is of $p$-character). The minima appear for photon energies where the $n p \rightarrow d$ transition-matrix element changes sign. The cross section from the outermost $s$-orbital is strongly coupled to this transition, leading to a "replica" of the Cooper minimum, sometimes called an induced Cooper minimum in the $n s \rightarrow p$ channels. Precisely at the photon energy for these cross-section minima, the two uppermost panels in Fig. 1 show delay maxima with peaks of several hundreds of attoseconds. Argon has been studied experimentally in this energy region [8,23], and Ref. [23] explains the absence of a large peak in the data with the dominance of shake-up channels, with a different delay, at energies where the direct ionization from $3 s$ vanishes. It is a general trend that the photoionization delay grows very large when the amplitude for the process goes to zero, as will be seen below.

For a pure Coulomb system, the contribution from an IR-photon, exchanged when the electron already is in the continuum, can be described with an analytical expression [35,36]. In Ref. [26] it was shown that its results agree remarkably well with many-body calculations of the difference between the atomic and the Wigner delay, at least for not too slow electrons emitted along the polarization axis. This was further shown to hold for a range of elements and orbitals. 

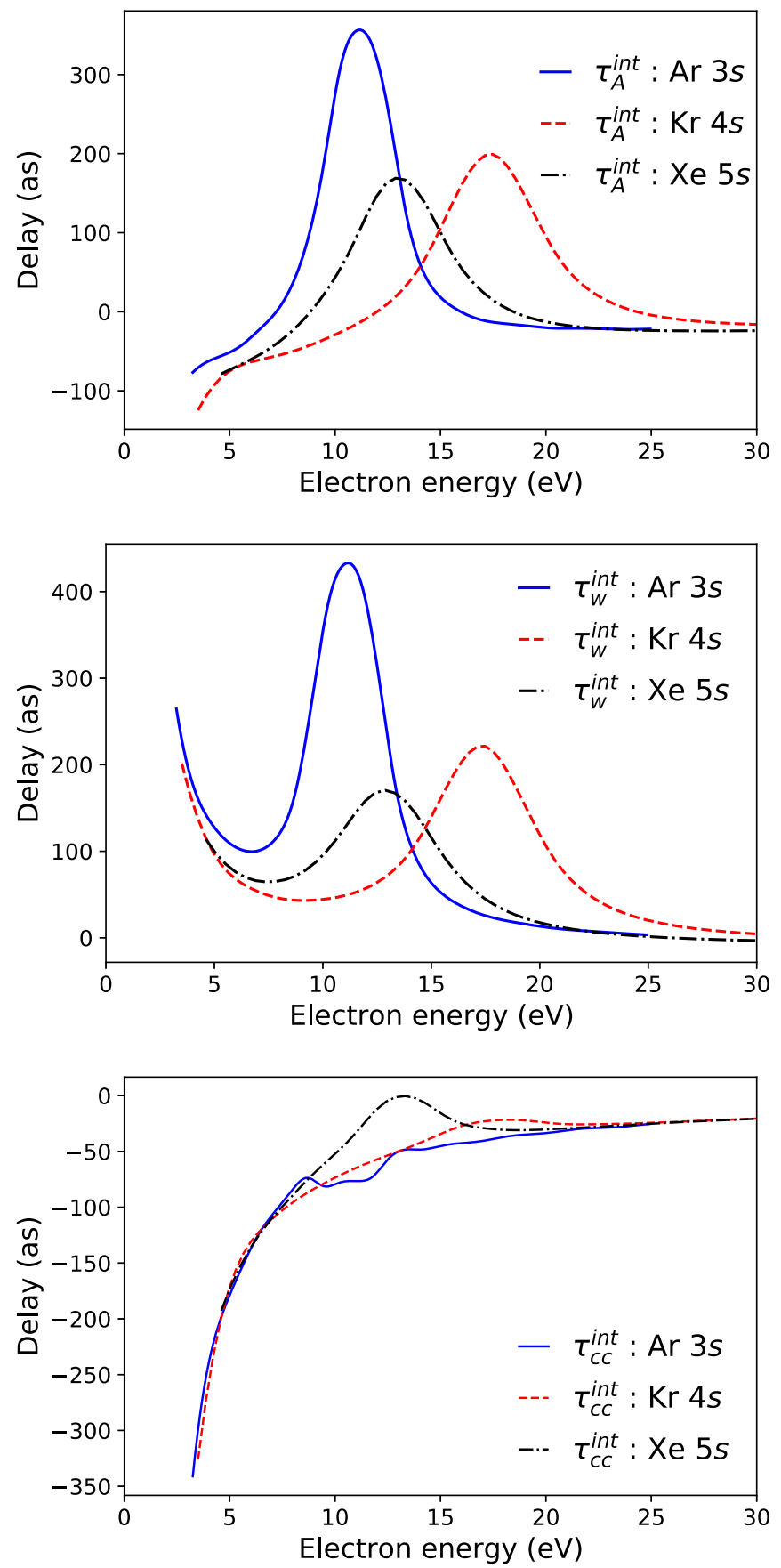

FIG. 1. The delay obtained when photoelectrons emitted in all angles are collected. Results are shown for electrons ionized from $\operatorname{argon} 3 s$, krypton $4 s$, and xenon $5 s$. The top panel shows the atomic delay [cf. Eqs. (34) and (35)], the middle panel shows the Wigner delay [cf. Eq. (32)], and the bottom panel shows the difference between them.

The difference between the atomic delay and the Wigner delay is generally largest for low photoelectron energies where the phases acquired in the absorption and the emission paths differ the most, and it approaches monotonically zero for larger and larger photoelectron energies. This is seen in the bottom panel in Fig. 1, but in addition there are distinct features around the induced Cooper minima which are specific for each element.
Below we will see that this is a property of the integrated signal, but that the universality of the cc-delay still does hold for a range of angles.

We now proceed to investigate the angular variation.

\section{B. Angular dependence}

Argon is essentially a nonrelativistic system. We thus expect the one-photon delay from an $s$-orbital to be more or less angular-independent, implying that $\tilde{\beta}_{2}^{W}$ in Eq. (33) is essentially real. That this is indeed the case is shown in the right panel of Fig. 2. The real part of $\tilde{\beta}_{2}^{W}$ is close to 2 , as we expect nonrelativistically, and the imaginary part is very small. Only at the induced Cooper minimum can small deviations from this be observed.

The $\tilde{\beta}$ 's for the two-photon process are displayed in the left panel of Fig. 2. Again we can note the expected nonrelativistic result discussed in Sec. II C with the real part of $\tilde{\beta}_{2}$ being close to $20 / 7$ and that of $\tilde{\beta}_{4}$ close to $8 / 7$, both with small imaginary parts. Only for low kinetic energies can a nonzero imaginary part be clearly seen.

Figure 3 displays the argon results for the differences between the delay as measured in a given angle and that for angular integrated measurements, for the atomic delay as well as for the underlying Wigner delay. The latter, which is a pure relativistic effect, is only of the order of a few attoseconds, and its energy dependency follows closely the imaginary part of $\tilde{\beta}_{2}^{W}$. This is expected since the variations in the real part of $\tilde{\beta}_{2}^{W}$ are only a few percent. At the so called magic angle $(\theta \approx 54.7)$, where $P_{2}(\cos \theta)=0$, the Wigner delay is identical to $\tau_{W}^{\text {int }}$. The magic angle result for the atomic delay (upper left panel of Fig. 3) is thus solely coming from the second photon. The difference between the atomic and the Wigner delay, for $\tau-\tau^{\text {int }}$, is displayed in the upper right panel; it differs only slightly from the atomic delay in the upper left panel showing that the angular dependence for argon is dominated by two-photon effects.

The heavier systems krypton and xenon, cf. Fig. 4, still show real parts of $\tilde{\beta}_{2}$ 's just below 3 and of $\tilde{\beta}_{4}$ 's just above 1 , but the modulations close to the Cooper minima get increasingly more pronounced as shown in the magnified middle panels. The deviation from $\tilde{\beta}_{2}^{W}=2$ is also increasing significantly, signaling the presence of an angular dependence already at the one-photon level.

The resulting differences between the delay as measured in a given angle, and that for an angular integrated measurement, are displayed in Fig. 5 for krypton and xenon. The angular dependence at low energy is clearly a two-photon effect (absent in the Wigner delay), and rather similar in magnitude for the two elements, as expected for a nonrelativistic effect. The angular dependence around the induced Cooper minima, on the other hand, is growing rapidly with the nuclear charge (note the different scales in the right and left columns of Fig. 5), as expected from an effect of relativistic origin. Here both the atomic delay (top panels) and the Wigner delay (middle panels) show significant angular variations. It is striking that the difference between the two still is more or less constant over a wide range of angles, as seen in the bottom panels. To 

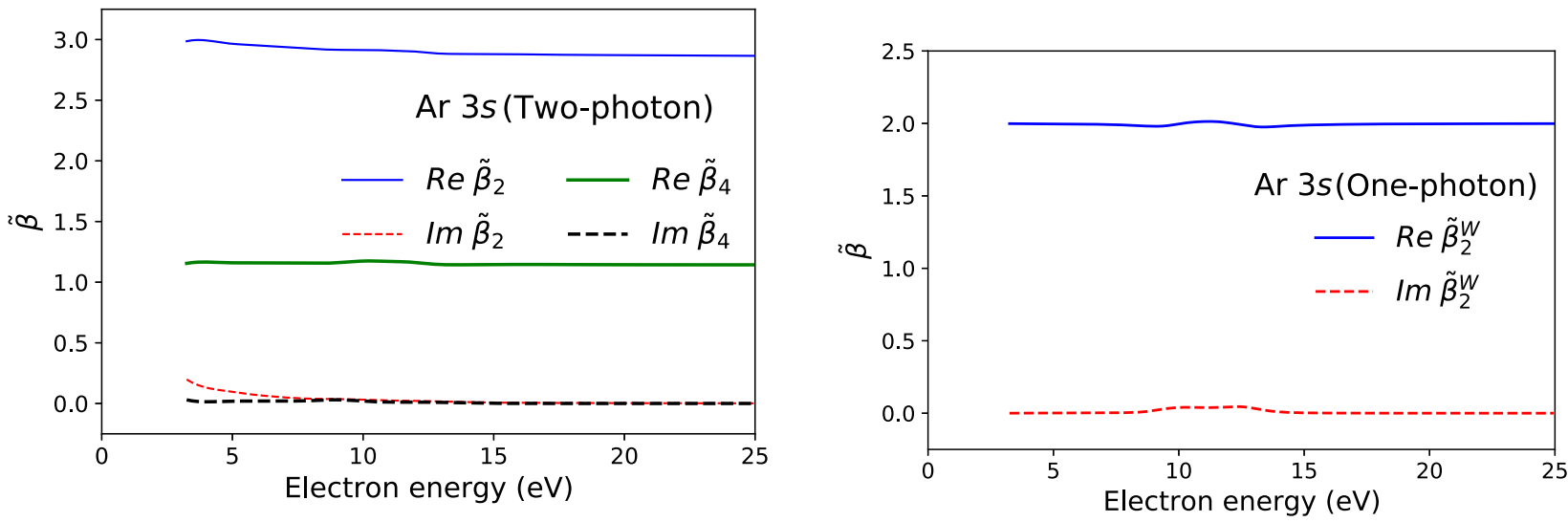

FIG. 2. Complex $\tilde{\beta}$ parameters for ionization from the $3 s$ subshell of argon. The left panel shows the real and imaginary parts of the complex $\tilde{\beta}_{2}$ and $\tilde{\beta}_{4}$ parameters (for the two-photon process). The right panel shows the real and imaginary parts of the complex $\tilde{\beta}_{2}^{W}$ parameter (for the one-photon process).

understand why, we note that the plotted quantity is

$$
\begin{array}{r}
\quad \Delta \tau_{A}-\Delta \tau_{W}=\tau_{A}(\theta)-\tau_{A}^{\mathrm{int}}-\left(\tau_{W}(\theta)-\tau_{W}^{\mathrm{int}}\right) \\
\rightarrow \tau_{A}(\theta)-\tau_{W}(\theta)=\Delta \tau_{A}-\Delta \tau_{W}+\left(\tau_{A}^{\mathrm{int}}-\tau_{W}^{\mathrm{int}}\right) .
\end{array}
$$

A closer comparison of the bottom panels in Fig. $5\left(\Delta \tau_{A}-\right.$ $\left.\Delta \tau_{W}\right)$ and the bottom panel in Fig. $1\left(\tau_{A}^{\mathrm{int}}-\tau_{W}^{\mathrm{int}}\right)$ shows that the structures close to the induced Cooper minima to some extent can cancel. This is seen in Fig. 6, which displays $\tau_{A}(\theta)-\tau_{W}(\theta)$ for some selected angles. Here just a tiny remnant of the variations at the Cooper minima can be seen. For small to modest angles, all the curves fall on top of each other, and they agree further with the analytical formula [36] down to $10 \mathrm{eV}$ electron kinetic energy. For larger angles, the
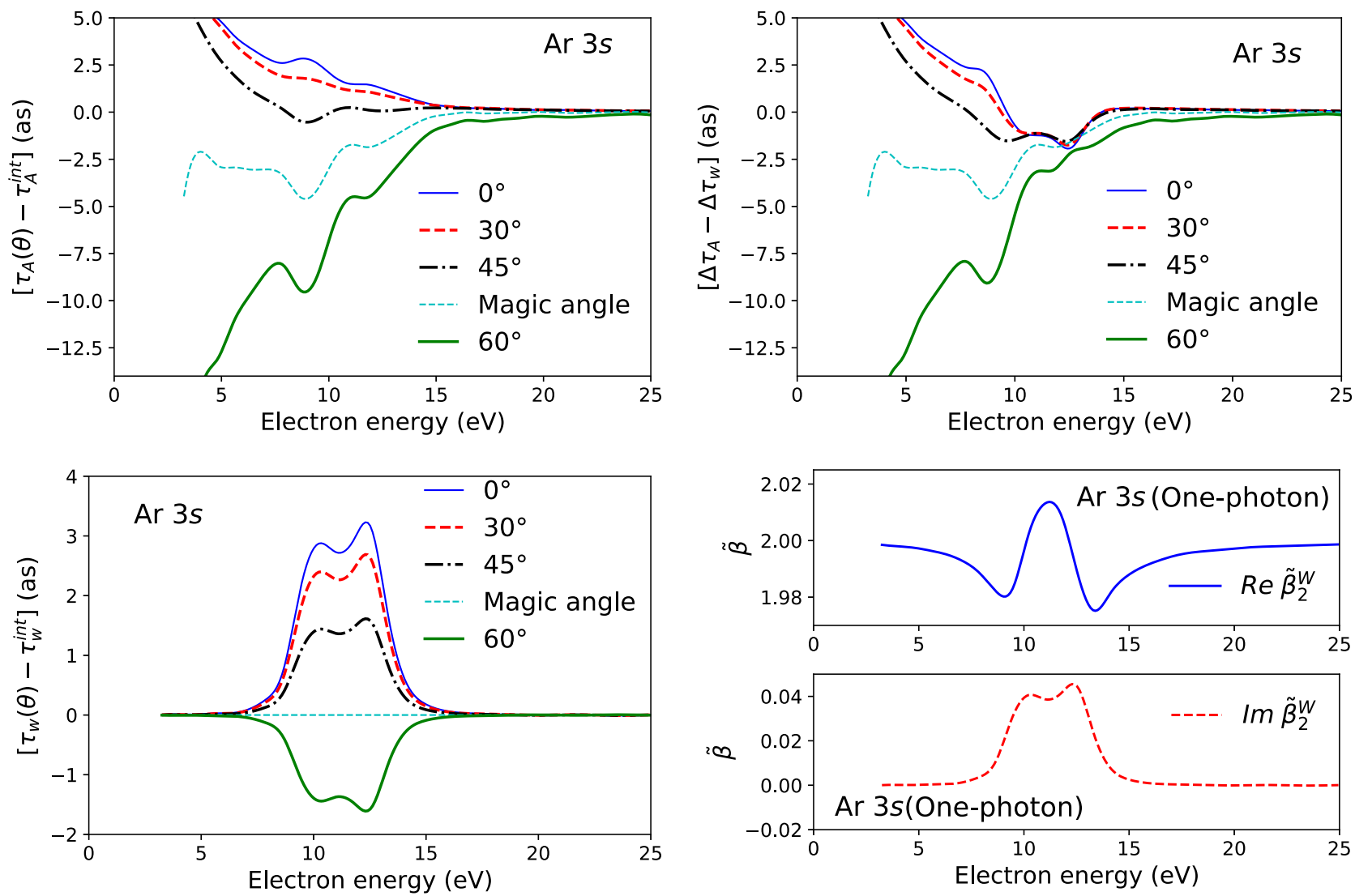

FIG. 3. The difference between the delay in a given angle and that from the integrated signal for ionization from the $3 s$-orbital in argon. The upper left panel shows this difference for the atomic delay. The lower left panel shows the same difference for the Wigner delay, while the upper right panel shows the difference between the previous two. The lower right panels show the real and imaginary parts of the $\tilde{\beta}_{2}^{W}$ parameter, same as in Fig. 2 (right panel), but on magnified $y$-scales. 

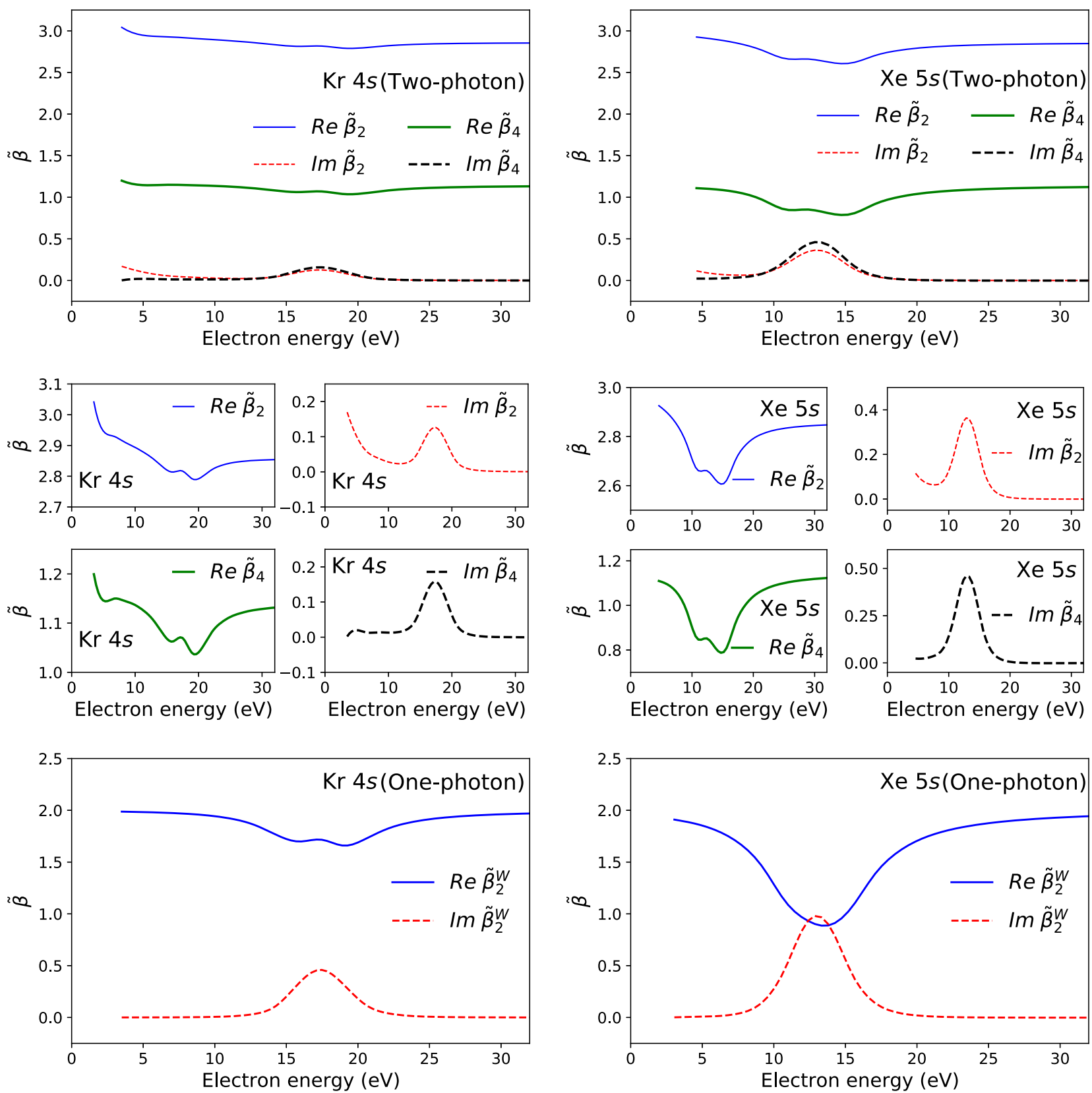

FIG. 4. $\tilde{\beta}$ parameters for krypton (left column) and xenon (right column). The top panels show the real and imaginary parts of the complex $\tilde{\beta}_{2}$ and $\tilde{\beta}_{4}$ parameters for two-photon ionization of the $4 s$ subshell of krypton (top left panel) and the $5 s$ subshell of xenon (top right panel), respectively. The middle panels show the same results as in top panels, but on magnified $y$-scales. The bottom panels show the real and imaginary parts of the complex $\tilde{\beta}_{2}$ parameter for one-photon ionization of the $4 s$ subshell of krypton (bottom left panel) and the $5 s$ subshell of xenon (bottom right panel), respectively.

curves show a different slope, but even then the two elements tend to follow each other closely. Only for the largest angles is there a real element-specific behavior. Experiments that directly compare the delay at different angles for not too low kinetic energies and not too large angles are thus rather insensitive to the contribution from the second photon, and this is true even in the region of an induced Cooper minimum. This is demonstrated in Fig. 7, where the atomic and Wigner delays of photoelectrons ejected at $30^{\circ}, 60^{\circ}$, and $70^{\circ}$ relative to the delay in the direction of the polarization of the light are shown. For not too small energies and not too large angles, the atomic and Wigner delays are very similar.

More insight into the angular dependence can be gained from Fig. 8, where the results from the left bottom panel in Fig. 5 (krypton $4 s$ ) are displayed as a function of angle. Figure 8 shows clearly the flat curve, essentially angular-independent, for small to modest angles, and the abrupt change after a certain critical angle. After that the delays quickly reach large negative values. This turning point is further moving outwards when the electron energy increases. 

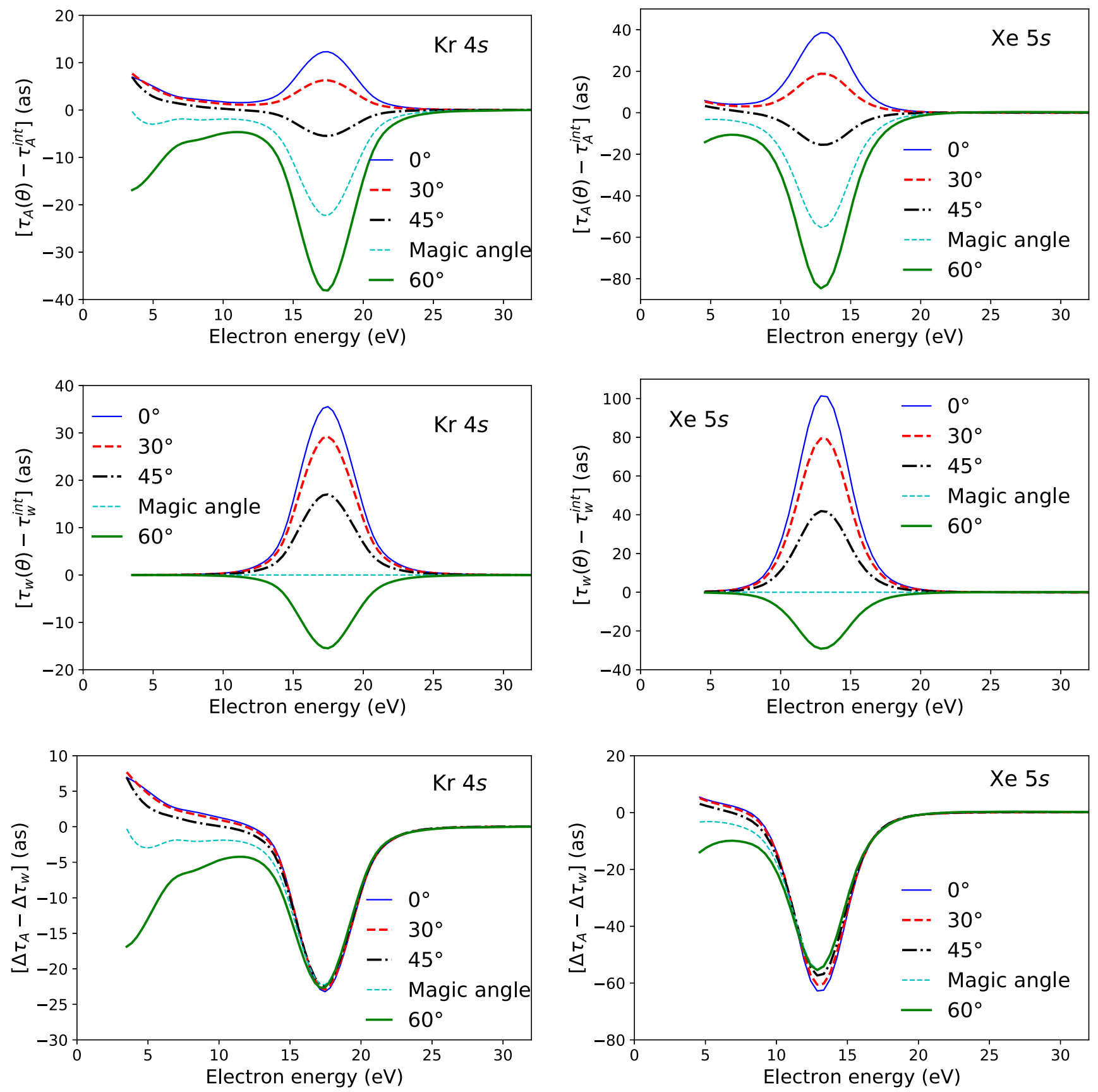

FIG. 5. The difference between the delay in a given angle and that from the integrated signal for ionization from krypton $4 s$ (left column) and xenon $5 s$ (right column). The top panels show this difference for the atomic delay, the middle panels show it for the Wigner delay, and the bottom panels show the difference between the two.

How can the large magnitudes of the delays at large angles be understood? The photoelectron wave function after absorption of the XUV photon has $p$-character, and thus a node at $90^{\circ}$. A soft IR-photon has further only a limited ability to alter the direction of the photoelectron, an ability that will decrease with increasing photoelectron energy. The $\beta$-parameters governing the angular dependence of the cross section, Eq. (1), will with increasing energy approach values such that $1+\beta_{2} P_{2}(\cos \pi / 2)+\beta_{4} P_{4}(\cos \pi / 2) \rightarrow 0$. The real part of each of the complex $\tilde{\beta}$-parameters is a kind of average of the regular $\beta$ 's for the cross section for the absorption and emission paths, respectively, as seen in particular for the higher energies in Fig. 9, and thus the real part of $1+\tilde{\beta}_{2} P_{2}(\cos \pi / 2)+\tilde{\beta}_{4} P_{4}(\cos \pi / 2)$ quickly approaches zero for all but the lowest energies. For the imaginary part of the expression, the situation is different. The imaginary parts for each of the $\tilde{\beta}$ 's do approach zero for higher energies but still somewhat slower than the real parts approach the values that cancel the cross section at $90^{\circ}$, leading to a quickly growing phase when this angle is approached. In Fig. 9 it is further seen how the Cooper minimum modulations of the $\beta$-parameters appear for higher electron energies in the absorption path compared to the emission patch, resulting in a double-well structure for $\tilde{\beta}$. 


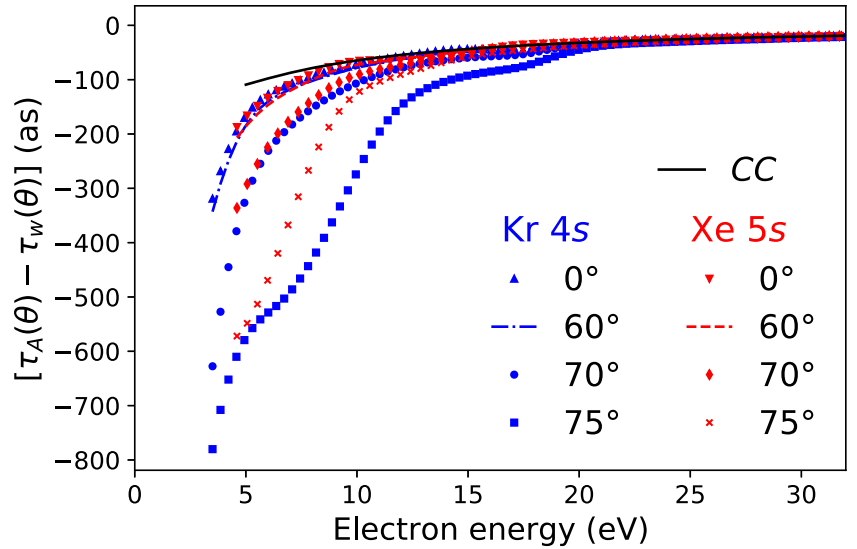

FIG. 6. The difference between the atomic delay and the Wigner delay for detection at a range of selected angles, for ionization from $\mathrm{Kr} 4 s$ and $\mathrm{Xe} 5 s$. The black line shows the analytical result from Ref. [36].

\section{CONCLUSION}

We have shown that the angular dependence of the photoionization delay can be described by complex anisotropy parameters. These parameters are closely related to the wellknown $\beta$-parameters used for many decades to characterize the angular distribution of photoelectron spectra, but since the delay-parameters depend on at least two quantum paths to a specific final state, they are inherently complex. Equipped with the parameters for one- and two-photon exchange, the angle dependence of the photoionization delay in RABBIT experiments can be efficiently analyzed.

We have further applied these anisotropy parameters to photoionization from the outermost $s$-orbital in some rare-gas atoms. Here the one-photon delay is angular-independent in a nonrelativistic framework, but the measurable two-photon delay does permit an angular dependence, as does the onephoton delay when relativistic effects are taken into account. This allows for an interesting interplay between two-photon effects and relativistic contributions, where the relative importance varies strongly when different elements are considered.
This situation could be conveniently analyzed with the complex anisotropy parameters.

Figure 7 indicates that, for energies close to an induced Cooper minimum, it should be possible to measure the delay at certain angles relative to a fixed angle and thereby be directly sensitive to the Wigner delay, which is intriguing. However, one should be aware that the large delays predicted by theory [9,25-27] close to the induced Cooper minima in many of the rare gases have not yet been seen in experiments. With the recently achieved improved energy resolution, experiments have instead highlighted the importance of shake-up channels [14,23]: in rare-gas atoms, channels of the type $n s^{2} n p^{4} n^{\prime} \ell^{\prime} \epsilon_{\ell^{\prime \prime}}$ can be populated by some teeth in the comb of harmonics, used in RABBIT experiments, and the ionized electrons might emerge with the same energy as the electrons in the usually dominating $n s n p^{6} \epsilon_{\ell}$ channel, ionized by another harmonic. In neon it was possible to disentangle the different channels to obtain results for the true delay when the photoelectron leaves the ion in the $2 s 2 p^{6}$ state [14], and in addition it was demonstrated that the delay of the photoelectron in the different channels can be distinctly different. When the cross section for the $n s n p^{6} \epsilon_{\ell}$ channel goes to zero at an induced Cooper minimum, however, the shake-up channels might dominate and such a disentanglement gets very hard. It is an open question whether there is a way around this.

\section{ACKNOWLEDGMENTS}

Support from the Swedish Research Council (Grant No. 2020-03315), the Knut and Alice Wallenberg Foundation (Grant No. 2017.0104), and the Wenner-Gren Foundation (Contract No. UPD2019-0095) is gratefully acknowledged.

\section{APPENDIX: EFFECTIVE TWO-PHOTON OPERATORS}

The interaction of an electromagnetic field within the dipole approximation is described by a tensor operator, $\mathbf{t}^{k}$ of rank $1(k=1)$ and odd parity. The components give the polarization, with $q=0$ corresponding to linearly polarized light, and $q= \pm 1$ corresponding to right-and left-handed circularly polarized light, respectively. The two-photon interaction is
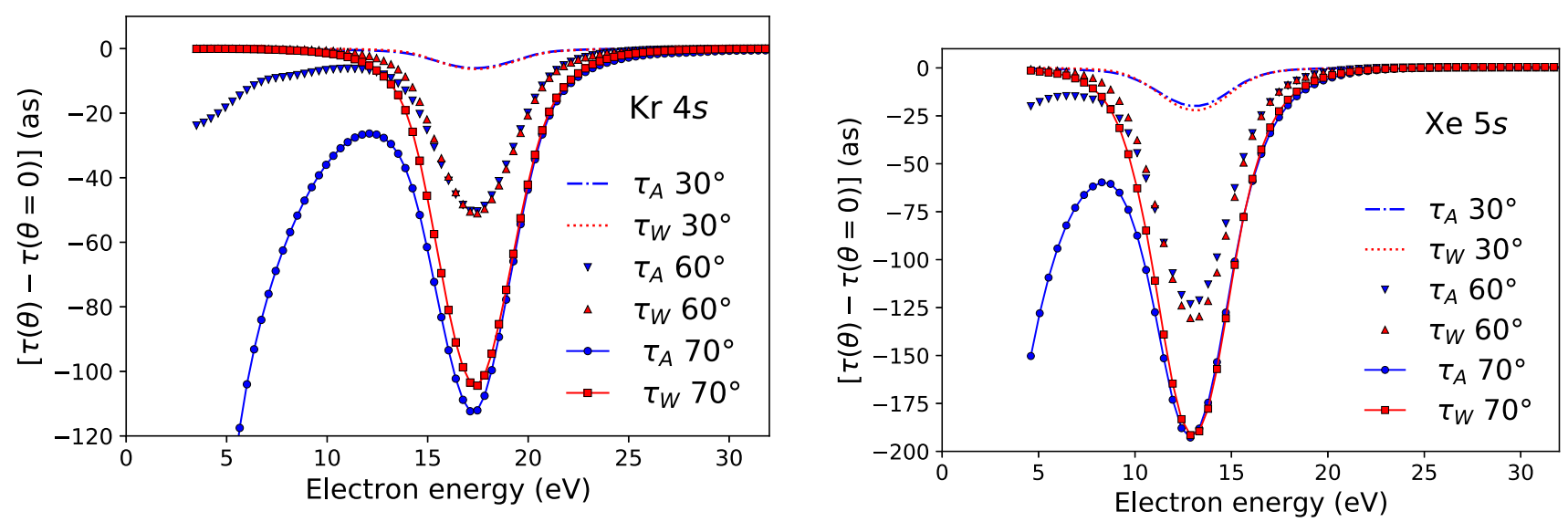

FIG. 7. The atomic and Wigner delays of photoelectrons ejected at angles $30^{\circ}, 60^{\circ}$, and $70^{\circ}$ relative to the delay in the direction of the polarization of the light, i.e., for $0^{\circ}$. For not too small energies and not too large angles, they are very similar. 


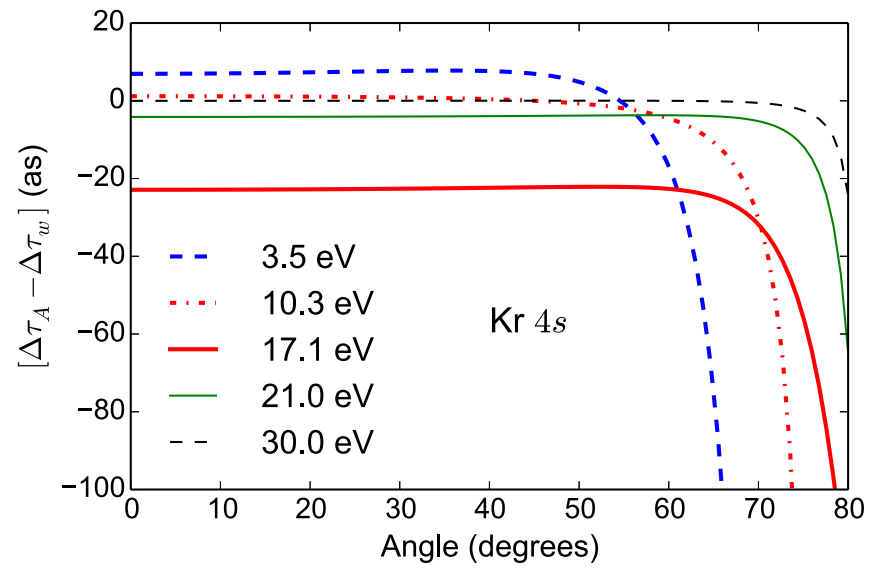

FIG. 8. The difference $\Delta \tau_{A}-\Delta \tau_{W}$ (cf. the bottom panels in Fig. 5) for krypton $4 s$ plotted as a function of angle for a few selected photoelectron energies.

due to two such operators and it is convenient to describe the total interaction in terms of effective two-photon operators. They will be of even parity and can have rank 0,1 , or 2 .

We note first that interaction with components $t_{q_{1}}^{k}$ and $t_{q_{2}}^{k}$ can bring an electron from an orbital with angular momentum $j m$ to one with angular momentum $j^{\prime} m^{\prime}$, where $m^{\prime}=m+$ $q_{1}+q_{2}$ via intermediate states with angular momentum $j^{\prime \prime} m^{\prime \prime}$, and use standard angular momentum theory, detailed, e.g., in [37], to obtain the effective operators of rank $K$. With $k=1$ the possible values of $K$ is $K=0,1,2$, although $K=1$ is not possible for linearly polarized light, i.e., when $q_{1}=q_{2}=0$. We have thus

$$
\begin{aligned}
& \sum_{\gamma^{\prime \prime}, j^{\prime \prime}, m^{\prime \prime}=m+q_{1}}\left\langle\gamma^{\prime} j^{\prime} m^{\prime}\left|t_{q_{2}}^{k}\right| \gamma^{\prime \prime} j^{\prime \prime} m^{\prime \prime}\right\rangle\left\langle\gamma^{\prime \prime} j^{\prime \prime} m^{\prime \prime}\left|t_{q_{1}}^{k}\right| \gamma j m\right\rangle \\
& =\sum_{\gamma^{\prime \prime}, j^{\prime \prime}}\left[\left\langle\gamma^{\prime} j^{\prime}|| \mathbf{t}^{k}|| \gamma^{\prime \prime} j^{\prime \prime}\right\rangle\left\langle\gamma^{\prime \prime} j^{\prime \prime}|| \mathbf{t}^{k}|| \gamma j\right\rangle\right. \\
& \times \sum_{m^{\prime \prime}=m+q_{1}, m^{\prime}=m^{\prime \prime}+q_{2}}(-1)^{j^{\prime}+j^{\prime \prime}-m^{\prime}-m^{\prime \prime}}\left(\begin{array}{ccc}
j^{\prime} & k & j^{\prime \prime} \\
-m^{\prime} & q_{2} & m^{\prime \prime}
\end{array}\right) \\
& \left.\times\left(\begin{array}{ccc}
j^{\prime \prime} & k & j \\
-m^{\prime \prime} & q_{1} & m
\end{array}\right)\right] \\
& =\sum_{K, \gamma^{\prime \prime}, j^{\prime \prime}, Q=q_{1}+q_{2}}(-1)^{j+j^{\prime}+K}(2 K+1) \\
& \times\left\langle\gamma^{\prime} j^{\prime}\left\|\mathbf{t}^{k}\right\| \gamma^{\prime \prime} j^{\prime \prime}\right\rangle\left\langle\gamma^{\prime \prime} j^{\prime \prime}\left\|\mathbf{t}^{k}\right\| \gamma j\right\rangle\left\{\begin{array}{ccc}
k & k & K \\
j & j^{\prime} & j^{\prime \prime}
\end{array}\right\} \\
& \times(-1)^{j^{\prime}-m^{\prime}}\left(\begin{array}{ccc}
j^{\prime} & K & j \\
-m^{\prime} & Q & m
\end{array}\right)(-1)^{-Q}\left(\begin{array}{ccc}
k & k & K \\
q_{2} & q_{1} & -Q
\end{array}\right),
\end{aligned}
$$

where $\gamma$ denotes all other quantum numbers of the orbital. The first equality in Eq. (A1) is easily obtained through the Wigner-Eckart theorem, and the second relies on the properties of the $3 j$ - and $6 j$-symbols.
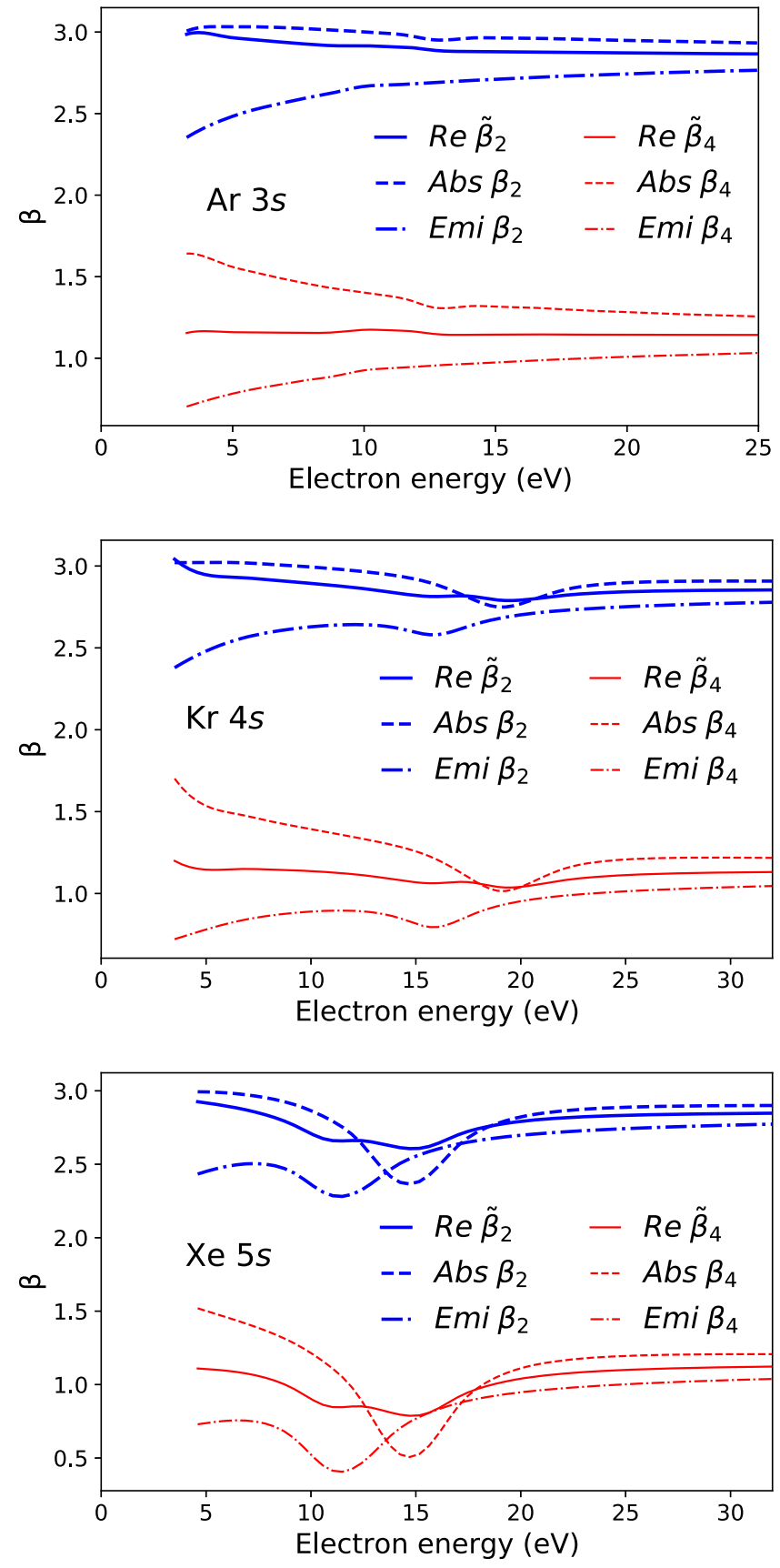

FIG. 9. The ordinary $\beta$ parameters (i.e., the parameters that determine the angle dependence of the cross section) for the RABBIT absorption path (dashed line), and the emission path (dashed-dotted line), compared to the real part of the complex $\tilde{\beta}$ parameters (solid line) for argon $3 s$ (top panel), krypton $4 s$ (middle panel), and xenon $5 s$ (bottom panel). The thick blue lines show the results for the $\beta_{2}$ and $\tilde{\beta}_{2}$ parameters, while the red thin lines show the results for the $\beta_{4}$ and $\tilde{\beta}_{4}$ parameters.

The dipole operator is in length gauge $e \mathbf{r}^{k}=\operatorname{er} \mathbf{C}^{1}$, where $\mathbf{C}^{k}$ denotes the tensor-operator form of the spherical harmonics, as introduced by Racah [38]. For this particular case, we 
may then write the two-photon matrix, Eq. (A1), as

$$
\begin{aligned}
\left\langle\gamma^{\prime} j^{\prime} m^{\prime}\left|X_{Q}^{K}\right| \gamma j m\right\rangle= & (-1)^{j^{\prime}-m^{\prime}}\left(\begin{array}{ccc}
j^{\prime} & K & j \\
-m^{\prime} & Q & m
\end{array}\right)(-1)^{j+j^{\prime}+K-Q}(2 K+1)\left(\begin{array}{ccc}
1 & 1 & K \\
q_{2} & q_{1} & -Q
\end{array}\right) \\
& \times \sum_{j^{\prime \prime}}\left\langle j^{\prime}\left\|\mathbf{C}^{1}\right\| j^{\prime \prime}\right\rangle\left\langle j^{\prime \prime}\left\|\mathbf{C}^{1}\right\| j\right\rangle R_{\gamma j \rightarrow \gamma^{\prime \prime} j^{\prime \prime} \rightarrow \gamma^{\prime} j^{\prime}}\left\{\begin{array}{ccc}
1 & 1 & K \\
j & j^{\prime} & j^{\prime \prime}
\end{array}\right\} \\
= & (-1)^{j^{\prime}-m^{\prime}}\left(\begin{array}{ccc}
j^{\prime} & K & j \\
-m^{\prime} & Q & m
\end{array}\right)\left\langle\gamma^{\prime} j^{\prime}\left\|X^{K}\right\| \gamma j\right\rangle,
\end{aligned}
$$

where $R$ denotes the radial integrals. Equation (A2) is independent of the intermediate $m$-values and the last equality defines the reduced matrix element, independent of all $m$-values, denoted by $\mathrm{M}$ above. In the relativistic coupling scheme, it is labeled by the $\kappa$-quantum-number of the photoelectron and the rank of the operator as

$$
\mathbf{M}_{\kappa, K}=(-1)^{j+j^{\prime}+K-Q}(2 K+1)\left(\begin{array}{ccc}
1 & 1 & K \\
q_{2} & q_{1} & -Q
\end{array}\right) \sum_{j^{\prime \prime}}\left\langle j^{\prime}\left\|\mathbf{C}^{1}\right\| j^{\prime \prime}\right\rangle\left\langle j^{\prime \prime}\left\|\mathbf{C}^{1}\right\| j\right\rangle R_{\gamma j \rightarrow \gamma^{\prime \prime} j^{\prime \prime} \rightarrow \gamma^{\prime} j^{\prime}}\left\{\begin{array}{lll}
1 & 1 & K \\
j & j^{\prime} & j^{\prime \prime}
\end{array}\right\} .
$$

It is sometimes convenient to include the phase of the outgoing electron in the matrix element:

$$
\overline{\mathrm{M}}_{\kappa, K}=\mathrm{M}_{\kappa, K} e^{i \Delta_{\kappa}} .
$$

We will in the following treat linearly polarized light only, implying that $q_{1}=q_{2}=Q=0$, and $m=m^{\prime}$. Further, if ionized from an $s$-orbital the photoelectron will after interaction with two photons be either of $s$ or $d$ character. With linearly polarized light only $K=0$ can contribute in the former case and only $K=2$ in the latter. This is true both for integer $j$ 's (the nonrelativistic coupling scheme) and for half-integer $j$ 's (the relativistic coupling scheme). In the nonrelativistic coupling scheme we have thus, for the particular case studied here, the angular contributions

$$
\begin{aligned}
\mathrm{M}_{\ell_{f}=K}= & (2 K+1)\left(\begin{array}{ccc}
1 & 1 & K \\
0 & 0 & 0
\end{array}\right) \\
& \times\left\langle K\left\|\mathbf{C}^{1}\right\| 1\right\rangle\left\langle 1\left\|\mathbf{C}^{1}\right\| 0\right\rangle\left\{\begin{array}{ccc}
1 & 1 & K \\
0 & K & 1
\end{array}\right\} R_{n s \rightarrow p \rightarrow \ell_{f}}
\end{aligned}
$$

$$
=\begin{aligned}
& \frac{1}{3} R_{n s \rightarrow p \rightarrow s} \text { for } K=0 \\
& \frac{2}{3} R_{n s \rightarrow p \rightarrow d} \text { for } K=2
\end{aligned}
$$

while the relativistic coupling scheme (with $j_{0}=1 / 2, \ell_{0}=0$, and $\ell_{f}=K$ ) gives

$$
\begin{aligned}
\mathbf{M}_{\kappa, K}= & (2 K+1)\left(\begin{array}{ccc}
1 & 1 & K \\
0 & 0 & 0
\end{array}\right) \\
& \times \sum_{j_{i}}(-1)^{j_{f}+1 / 2}\left\langle j_{f}\left\|\mathbf{C}^{1}\right\| j_{i}\right\rangle\left\langle j_{i}\left\|\mathbf{C}^{1}\right\| \frac{1}{2}\right\rangle \\
& \times\left\{\begin{array}{ccc}
1 & 1 & K \\
\frac{1}{2} & j_{f} & j_{i}
\end{array}\right\} R_{n s_{1 / 2} \rightarrow p_{j_{i}} \rightarrow \ell_{j_{f}}} .
\end{aligned}
$$

[1] J. Cooper and R. N. Zare, J. Chem. Phys. 48, 942 (1968).

[2] M. Y. Amusia, Atomic Photoeffect (Plenum, New York, 1990).

[3] K. L. Reid, Annu. Rev. Phys. Chem. 54, 397 (2003).

[4] M. S. Child, Phys. Chem. Chem. Phys. 10, 6169 (2008).

[5] P. M. Paul, E. S. Toma, P. Breger, G. Mullot, F. Augé, P. Balcou, H. G. Muller, and P. Agostini, Science 292, 1689 (2001).

[6] J. Itatani, F. Quéré, G. L. Yudin, M. Y. Ivanov, F. Krausz, and P. B. Corkum, Phys. Rev. Lett. 88, 173903 (2002).

[7] M. Schultze, M. Fiess, N. Karpowicz, J. Gagnon, M. Korbman, M. Hofstetter, S. Neppl, A. L. Cavalieri, Y. Komninos, T. Mercouris et al., Science 328, 1658 (2010).

[8] K. Klünder, J. M. Dahlström, M. Gisselbrecht, T. Fordell, M. Swoboda, D. Guénot, P. Johnsson, J. Caillat, J. Mauritsson, A. Maquet et al., Phys. Rev. Lett. 106, 143002 (2011).

[9] D. Guénot, K. Klünder, C. L. Arnold, D. Kroon, J. M. Dahlström, M. Miranda, T. Fordell, M. Gisselbrecht, P. Johnsson, J. Mauritsson et al., Phys. Rev. A 85, 053424 (2012).
[10] D. Guénot, D. Kroon, E. Balogh, E. W. Larsen, M. Kotur, M. Miranda, T. Fordell, P. Johnsson, J. Mauritsson, M. Gisselbrecht et al., J. Phys. B 47, 245602 (2014).

[11] M. Sabbar, S. Heuser, R. Boge, M. Lucchini, T. Carette, E. Lindroth, L. Gallmann, C. Cirelli, and U. Keller, Phys. Rev. Lett. 115, 133001 (2015).

[12] M. Kotur, D. Guénot, A. Jimenez-Galan, D. Kroon, E. W. Larsen, M. Louisy, S. Bengtsson, M. Miranda, J. Mauritsson, C. L. Arnold et al., Nat. Commun. 7, 10566 (2016).

[13] V. Gruson, L. Barreau, Á. Jiménez-Galan, F. Risoud, J. Caillat, A. Maquet, B. Carré, F. Lepetit, J.-F. Hergott, T. Ruchon et al., Science 354, 734 (2016).

[14] M. Isinger, R. J. Squibb, D. Busto, S. Zhong, A. Harth, D. Kroon, S. Nandi, C. L. Arnold, M. Miranda, J. M. Dahlström et al., Science 358, 893 (2017).

[15] C. Cirelli, C. Marante, S. Heuser, C. Petersson, Á. J. Galán, L. Argenti, S. Zhong, D. Busto, M. Isinger, S. Nandi et al., Nat. Commun. 9, 955 (2018). 
[16] C. Palatchi, J. M. Dahlström, A. S. Kheifets, I. A. Ivanov, D. M. Canaday, P. Agostini, and L. F. DiMauro, J. Phys. B 47, 245003 (2014).

[17] S. Heuser, A. Jiménez Galán, C. Cirelli, C. Marante, M. Sabbar, R. Boge, M. Lucchini, L. Gallmann, I. Ivanov, A. S. Kheifets et al., Phys. Rev. A 94, 063409 (2016).

[18] L. Eisenbud, Ph.D. thesis, Princeton University, 1948.

[19] E. P. Wigner, Phys. Rev. 98, 145 (1955).

[20] F. T. Smith, Phys. Rev. 118, 349 (1960).

[21] D. Busto, J. Vinbladh, S. Zhong, M. Isinger, S. Nandi, S. Maclot, P. Johnsson, M. Gisselbrecht, A. L'Huillier, E. Lindroth et al., Phys. Rev. Lett. 123, 133201 (2019).

[22] J. Joseph, F. Holzmeier, D. Bresteau, C. Spezzani, T. Ruchon, J. F. Hergott, O. Tcherbakoff, P. D’Oliveira, J. C. Houver, and D. Dowek, J. Phys. B 53, 184007 (2020).

[23] C. Alexandridi, D. Platzer, L. Barreau, D. Busto, S. Zhong, M. Turconi, L. Neoričić, H. Laurell, C. L. Arnold, A. Borot et al., Phys. Rev. Research 3, L012012 (2021).

[24] A. S. Kheifets, D. Toffoli, and P. Decleva, J. Phys. B 53, 115201 (2020).

[25] J. Vinbladh, J. M. Dahlström, and E. Lindroth, Phys. Rev. A 100, 043424 (2019).
[26] J. M. Dahlström, T. Carette, and E. Lindroth, Phys. Rev. A 86, 061402(R) (2012).

[27] J. M. Dahlström and E. Lindroth, J. Phys. B 47, 124012 (2014).

[28] V. Véniard, R. Taïeb, and A. Maquet, Phys. Rev. A 54, 721 (1996).

[29] M. Isinger, D. Busto, S. Mikaelsson, S. Zhong, C. Guo, P. Salières, C. L. Arnold, A. L'Huillier, and M. Gisselbrecht, Philos. Trans. R. Soc. A 377, 20170475 (2019).

[30] A. Maquet and R. Taïeb, J. Mod. Opt. 54, 1847 (2007).

[31] V. B. Beretskii, E. M. Lifshitz, and I. P. Pitaevskii, Relativistic Quantum Theory, 1st ed. (Pergamon, Oxford, 1971).

[32] W. R. Johnson and K. T. Cheng, J. Phys. B 12, 863 (1979).

[33] J. Vinbladh, Thesis, Stockholm University, 2019.

[34] J. W. Cooper, Phys. Rev. 128, 681 (1962).

[35] J. Dahlström, D. Guénot, K. Klünder, M. Gisselbrecht, J. Mauritsson, A. L'Huillier, A. Maquet, and R. Taïeb, Chem. Phys. 414, 53 (2013).

[36] J. M. Dahlström, A. L'Huillier, and A. Maquet, J. Phys. B 45, 183001 (2012).

[37] I. Lindgren and J. Morrison, Atomic Many-Body Theory, 2nd ed., Series on Atoms and Plasmas (Springer-Verlag, New York, 1986).

[38] G. Racah, Phys. Rev. 62, 438 (1942). 